\title{
Self-referenced spectral interferometry for single-shot measurement of sub-5-fs pulses
}

Cite as: Rev. Sci. Instrum. 86, 113106 (2015); https://doi.org/10.1063/1.4936289

Submitted: 30 July 2015 . Accepted: 10 November 2015 . Published Online: 23 November 2015

A. Trabattoni, T. Oksenhendler, H. Jousselin, G. Tempea, S. De Silvestri, G. Sansone, F. Calegari, and M. Nisoli

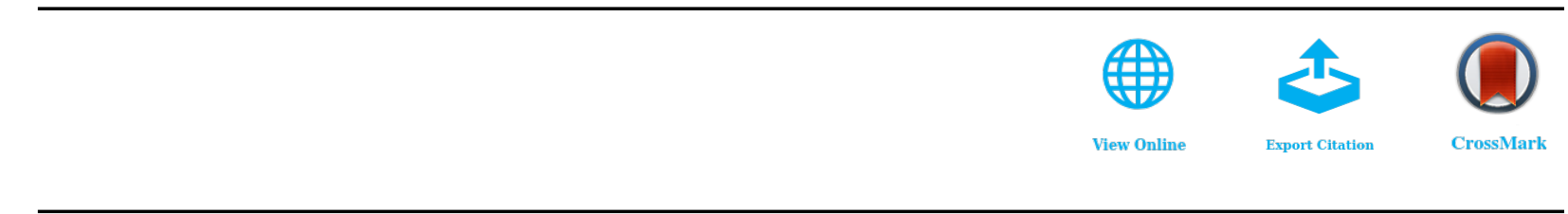

\section{ARTICLES YOU MAY BE INTERESTED IN}

Ultrafast optical parametric amplifiers

Review of Scientific Instruments 74, 1 (2003); https://doi.org/10.1063/1.1523642

Generation of high energy $10 \mathrm{fs}$ pulses by a new pulse compression technique Applied Physics Letters 68, 2793 (1996); https://doi.org/10.1063/1.116609

Measuring ultrashort laser pulses in the time-frequency domain using frequency-resolved optical gating

Review of Scientific Instruments 68, 3277 (1997); https://doi.org/10.1063/1.1148286

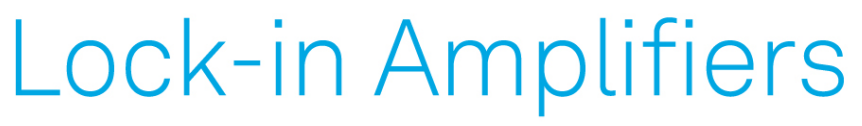

... and more, from DC to $600 \mathrm{MHz}$ Watch 


\title{
Self-referenced spectral interferometry for single-shot measurement of sub-5-fs pulses
}

\author{
A. Trabattoni, ${ }^{1, a)}$ T. Oksenhendler, ${ }^{2}$ H. Jousselin, ${ }^{2}$ G. Tempea, ${ }^{3}$ S. De Silvestri, ${ }^{1,4}$ \\ G. Sansone,,$^{1,4}$ F. Calegari, ${ }^{4}$ and M. Nisoli ${ }^{1,4}$ \\ ${ }_{1}^{1}$ Department of Physics, Politecnico di Milano, Piazza Leonardo da Vinci 32, 20133 Milano, Italy \\ ${ }^{2}$ Fastlite, Campus Scientifique d'Orsay, Bât 503, Plateau du Moulon, 91400 Orsay, France \\ ${ }^{3}$ Femtolasers Produktions GmbH, Fernkorngasse 10, 1100 Vienna, Austria \\ ${ }^{4}$ Institute of Photonics and Nanotechnologies, IFN-CNR, Piazza Leonardo da Vinci 32, 20133 Milano, Italy
}

(Received 30 July 2015; accepted 10 November 2015; published online 23 November 2015)

\begin{abstract}
We demonstrate a novel approach for the extension of self-referenced spectral interferometry to the temporal characterization of few-optical cycle pulses. The new experimental setup is characterized by low dispersion and a collinear geometry. 4-fs pulses have been characterized by performing single-shot measurements, with high dynamic range on a broad temporal region. An independent measurement of the pulse duration, obtained by using attosecond streaking, allowed us to cross-check the experimental technique. (ㅇ 2015 AIP Publishing LLC. [http://dx.doi.org/10.1063/1.4936289]
\end{abstract}

\section{INTRODUCTION}

Complete temporal characterization of femtosecond light pulses is of crucial importance for a number of experiments aiming to explore ultrafast phenomena. Several methods have been proposed and demonstrated to retrieve the amplitude and phase of ultrashort pulses. The most widely used techniques are Frequency Resolved Optical Gating (FROG) ${ }^{1,2}$ and Spectral Interferometry for Direct Electric-field Reconstruction (SPIDER) ${ }^{3-5}$ Various different implementations of these measurement schemes have been introduced in order to improve the performances, in terms of reliability, accuracy, temporal resolution, and dynamic range.

In 2010, a new technique for complete temporal characterization of ultrashort pulses has been introduced, named Self-Referenced Spectral Interferometry (SRSI). ${ }^{6}$ Unlike the previous methods, SRSI is based on the use of a reference pulse with a known spectral phase on a bandwidth larger than that of the pulse to be measured. This characteristic greatly simplifies the experimental scheme and the retrieval algorithm. ${ }^{7}$ This method is self-referenced since the reference pulse used for the measurement is generated from the pulse itself. Indeed, the reference is obtained by temporally filtering the input pulse by using cross-polarized wave (XPW) generation, which is a degenerate four-wave mixing process obtained in a nonlinear medium with anisotropic third order nonlinear susceptibility. ${ }^{8,9}$ In this case, the input pulse is filtered by its own temporal intensity. Within the slowly varying envelope approximation, in the case of undepleted pump and thin XPW crystal, $E_{X}(t) \propto\left|E_{I}(t)\right|^{2} E_{I}(t)$, where $E_{I}(t)$ and $E_{X}(t)$ are the temporal amplitudes of the electric field of the input and reference XPW pulses, respectively. The XPW process leads to the generation of a wave with orthogonal polarization with respect to the linearly polarized input wave.

\footnotetext{
a) Author to whom correspondence should be addressed. Electronic mail: andrea.trabattoni@polimi.it
}

The experimental implementation of SRSI requires the generation of a weak replica of the pulse to be characterized, which is perpendicularly polarized and properly delayed with respect to the input pulse. The main portion of the pulse is then used for XPW generation in a nonlinear crystal, resulting in a time-filtered reference pulse. The interference between the reference pulse and the replica is recorded and analyzed by applying Fourier transform spectral interferometry (FTSI). ${ }^{9}$ This algorithm allows one to retrieve amplitude and phase of the input pulse, thus leading to a complete temporal characterization. In the case of input pulses with very low chirp, the spectrum of the XPW pulse is larger than that of the input pulse, with a strongly reduced spectral chirp, ${ }^{10}$ thus leading to the generation of an almost ideal reference pulse for FTSI. ${ }^{9}$ So far the SRSI technique has been applied to the characterization and fine compression of sub- $15 \mathrm{fs}$ pulses, yielding an accurately measured coherent contrast of $50 \mathrm{~dB}$ on a \pm 400 -fs time range. ${ }^{11}$

In this work, we report on the experimental demonstration that SRSI, implemented with a low-dispersion and collinear optical setup, can be used to measure the temporal characteristics of sub-5-fs light pulses on a single-shot basis, with high dynamic range. Pulses as short as 4 fs have been measured, with 40-dB dynamic range on a 1-ps temporal window. This result has been cross-checked by directly measuring the electric field of the few-optical cycle pulses, using the attosecond streakcamera technique. ${ }^{12,13}$ The excellent agreement between the two measurements demonstrates the possibility of exploiting SRSI for complete temporal characterization of few-optical cycle light pulses.

\section{EXPERIMENTAL SETUP}

The layout of the experimental setup is shown in Fig. 1. 25 -fs pulses, with an energy of $6 \mathrm{~mJ}$ and $1-\mathrm{kHz}$ repetition rate, were generated by a Ti:sapphire laser system (Femtopower V PRO CEP, Femtolasers GmbH). The carrier-envelope phase 


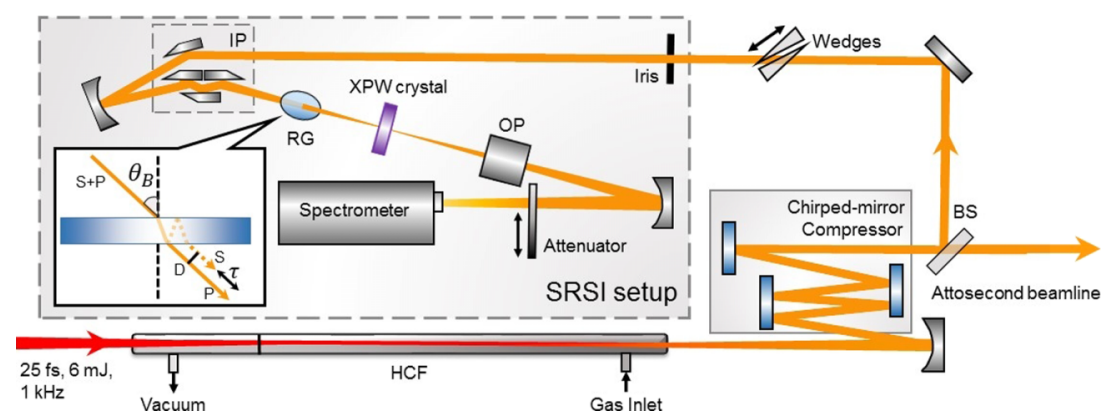

FIG. 1. Experimental setup: HCF is the hollow-core fiber, BS is a broadband beam splitter, IP is the input polarizer made by a combination of 4 GaAs plates, RG is the fused silica plate used for the generation of the pulse replica, OP is the output polarizer. The inset shows an enlarged view of the fused silica plate used for the generation of the temporally delayed weak replica of the input pulse.

(CEP) of the pulses was actively stabilized by using two feedback loops, with a residual fluctuation of about $200 \mathrm{mrad}$ (rms). The pulses were subsequently compressed by using the hollow fiber compression technique, ${ }^{14,15}$ in combination with broadband chirped mirrors (Mosaic OS, Femtolasers GmbH). The pulses were focused by a $1.75-\mathrm{m}$ focal length mirror into a 1-m-long hollow fiber, made of fused silica, with an inner diameter of $320 \mu \mathrm{m}$, filled with helium. A pressure gradient configuration was implemented ${ }^{16}$ to avoid nonlinear processes occurring before coupling the pulses into the hollow core fiber, with detrimental consequences on the performances of the compression setup in terms of coupling efficiency and CEP stability. ${ }^{17}$ The gas pressure inside the fiber increases from $<1$ mbar at the input, up to 2 bars at the output. The beam position of the input of the hollow fiber was actively stabilized. ${ }^{18}$ Pulse energy up to $2.3 \mathrm{~mJ}$ was obtained at the output of the chirped-mirror compressor.

A small portion of the beam, reflected by an uncoated fused-silica wedge, was sent in the SRSI setup, while the transmitted beam was directed in an extreme ultraviolet (XUV) beamline, designed for the generation and application of isolated attosecond pulses. ${ }^{19,20}$ A pair of fused silica ultra-thin wedges (apex angle $2^{\circ} 48^{\prime}$ ) at Brewster angle was placed at the input of the SRSI setup. Upon translating one of the two wedges, it was possible to achieve fine tuning of the pulse dispersion compensation. The total amount of fused silica in the beam path was adjusted in order to measure the shortest pulse duration with the SRSI setup. Taking advantage of the single-shot nature of the measurement technique, the best wedge position could be easily and rapidly obtained. The dashed box in Fig. 1 shows the SRSI optical setup, which was designed in order to have a collinear geometry for the reference and main pulses and a very low dispersion, which is a crucial requirement for the measurement, as it will be discussed in Sec. III. A polarizer is installed at the entrance, since XPW generation is particularly sensible to the quality of input polarization. The polarizer consists of a combination of reflective Brewster incidence GaAs plates, which introduce negligible dispersion. A weak replica of the pulse is generated by double internal reflection in a $120-\mu$ m-thick fused silica plate, oriented at Brewster incidence angle for $p$-polarization. The input polarizer is aligned in such a way that the polarization of the pulse at the input of the fused silica plate is not purely $p$-wave. The weak $s$-wave component is then reflected at each air-glass interface of the fused silica plate, thus leading to the generation of a replica of the main pulse, with a temporal delay, $\tau$, related to the plate thickness. For a $120-\mu$ m-thick plate, the delay between the $p$-wave main pulse and the $s$ wave weak replica pulse is $\tau \sim 1 \mathrm{ps}$. The contribution from other replicas generated by high-order internal reflections can be completely neglected. The lateral displacement of the pulse replica with respect to the main pulse transmitted by the plate was $\sim 94 \mu \mathrm{m}$. Particular attention was paid to the selection of a fused silica plate with almost perfectly parallel faces; indeed, small angles between the faces would result in an increase of the lateral displacement. Moreover, tight focusing of the beam onto the 10- $\mu \mathrm{m}$ input slit of the spectrometer used in the SRSI setup was avoided. The input polarizer and the fused silica plate introduced a dispersion of $\sim 5 \mathrm{fs}^{2}$ on the $p$-wave main pulse and $\sim 15 \mathrm{fs}^{2}$ on the pulse replica, due to the double internal reflection. This difference in the dispersion for the two pulses was taken into account in the calibration of the setup, so that it does not affect the quality and the validity of the measurement.

After the generation of the pulse replica, the reference pulse was produced by focusing the $p$-wave pulse in a $200-\mu \mathrm{m}$-thick $\mathrm{BaF}_{2}$ crystal for XPW generation. The reference pulse generated in the crystal was perpendicularly polarized with respect to the $p$-wave input pulse, on the same axis of the pulse replica, which propagates through the XPW crystal without any nonlinear effect. The XPW crystal introduced an additional dispersion of $7.5 \mathrm{fs}^{2}$. We note that for the generation of the reference pulse, the conversion efficiency of the XPW process is not important, while spectral broadening and flat spectral phase are of paramount importance. The measured conversion efficiency of the XPW process was $\sim 0.5 \%$.

Finally, the XPW reference pulse and the pulse replica with a temporal delay $\tau$ are selected by a polarizer and interfere into the spectrometer. We observe that in the framework of the standard FTSI processing, delay $\tau$ must be large enough to avoid any relevant overlap between the terms centered around $t=\tau$ and $t=0$ obtained by taking the inverse Fourier transform of the spectrum produced by the interference between the XPW reference pulse and the temporally delayed pulse replica. The upper limit is chosen such that the period of the spectral modulation imposed by the interference can be perfectly resolved by the spectrometer used in the experimental setup. Any additional dispersion after the XPW crystal is balanced and thus has no influence on the measurement. It is therefore possible to use a standard ultrabroadband calcite 
Glan-Thomson output polarizer. Due to the broad spectral bandwidth of the pulses to be measured, the spectrometer requires a precise calibration of its spectral intensity response. Furthermore, the retrieval procedure needs to take into account an intensity calibration on the fringe term of the SRSI signal and a spectral phase correction due to slight errors in the wavelength calibration. ${ }^{21}$ These calibrations can be performed by using an intensity calibrated white light. In the case of the experiment reported in this work, the detectable bandwidth ranges from $450 \mathrm{~nm}$ up to $970 \mathrm{~nm}$, which corresponds to a pulse transform-limited duration of $3.2 \mathrm{fs}$.

\section{RESULTS AND DISCUSSION}

We have first analyzed the validity range of the measurement by considering the convergence condition of the SRSI algorithm, which can be expressed in terms of the broadening factor, $\Omega=\Delta \omega_{X P W} / \Delta \omega_{0}$, where $\Delta \omega_{0}$ and $\Delta \omega_{X P W}$ are the rms bandwidths of the input pulse and of the reference XPW pulse, respectively. The measurement criterion required for the validity of the pulse characterization is given by: $\Omega>\Omega_{\text {lim }}$, where $\Omega_{\text {lim }}$ depends only on the spectral amplitude profile of the input pulse: ${ }^{22}$ in the case of Gaussian pulses, $\Omega_{\text {lim }}=1$. Such validity condition can be easily checked experimentally from the measurement of the spectral broadening of the reference pulse. In order to have spectral broadening of the XPW pulse (with corresponding pulse duration reduction), the second order spectral phase coefficient (chirp) of the input pulse, $\phi^{(2)}$, has to be very close to zero during the interaction. ${ }^{10}$ The maximum spectral broadening ( $\Omega=\sqrt{3}$ for Gaussian pulses) is obtained for input pulses with zero chirp. Therefore, to ensure the validity of the measurement technique, the chirp of the input pulses has to be minimized. It is instructive to write the convergence condition in terms of the relative chirp factor, $x$, of the input pulse, defined as $x=\phi^{(2)} / \Delta \tau_{0}^{2}$, where
$\Delta \tau_{0}$ is the rms duration of the input pulse. As a rule of thumb, the validity of the measurement is guaranteed if $|x| \leq \sqrt{3} / \alpha$, where $\alpha$ is the time-bandwidth product of the input pulse (in the case of zero chirp). ${ }^{22}$ Therefore, a 4-fs (full-width at half maximum (FWHM) duration, corresponding to 1.7 -fs rms duration) pulse can be measured if its chirp is smaller than $\sim 10 \mathrm{fs}^{2}$, so that it is already very close to its transformlimited value. For this reason, in the case of few-optical cycle pulses, the SRSI optical setup has to be designed in order to introduce less dispersion as possible, as described in Sec. II.

The spectrum of the pulse to be characterized is shown by the solid black line in Fig. 2(a) and extends from $480 \mathrm{~nm}$ up to $920 \mathrm{~nm}$. The grey dashed curve is the spectrum of the reference XPW pulse: the broadening in the XPW signal spectrum is more pronounced on the blue side in comparison with the red one, due to the frequency dependence of the XPW generation process. ${ }^{22}$ The measured spectral broadening of the XPW pulse is a first indication of the low chirp of the input pulses and can be used for a first optimization of the dispersion, by adjusting the amount of glass introduced by the fused silica wedges. We note that all the spectra were acquired on a singleshot basis. The retrieved temporal intensity profile of the pulse is reported in Fig. 2(b), while its spectral phase is shown as blue line in Fig. 2(a). The corresponding pulse duration (FWHM) was $4 \mathrm{fs}$, very close to the transform-limited value of 3.9 fs. Figure 2(c) shows the retrieved temporal intensity profile on a logarithmic scale, on a \pm 500 -fs temporal range, showing a 40-dB dynamic range. We note that, by using the SRSI method, single-shot characterization of few-cycle pulses can be obtained with a large dynamics range even by using a commercial spectrometer with modest signal-to-noise ratio. The temporal range of the measurement and the large dynamic range allowed us to estimate the pulse energy spread around the main peak (at $t=0$ ). We have calculated the standard deviation over the measured temporal range, $T=1 \mathrm{ps},{ }^{11}$
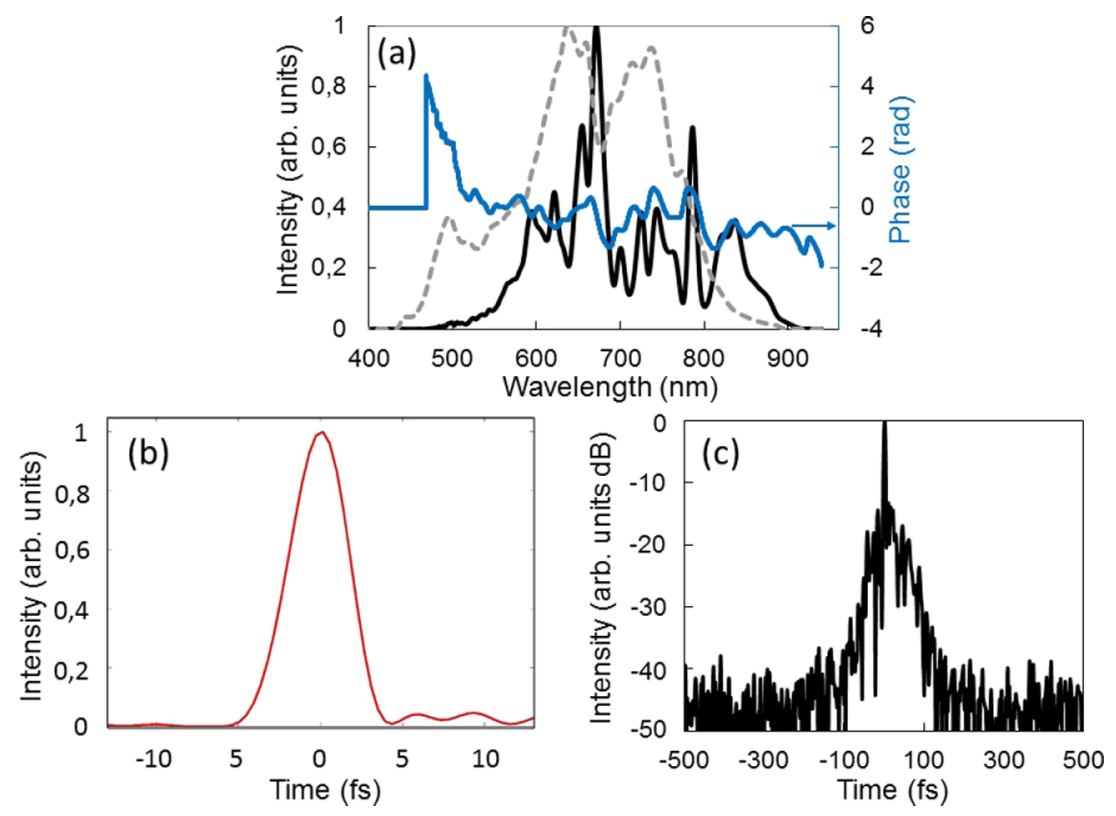

FIG. 2. (a) Spectral amplitudes of the input pulse (black line) and of the XPW reference pulse (dashed grey line). The blue line corresponds to the retrieved spectral phase of the input pulse. (b) Retrieved temporal intensity profile in linear scale. (c) Retrieved temporal intensity profile in logarithmic scale. 

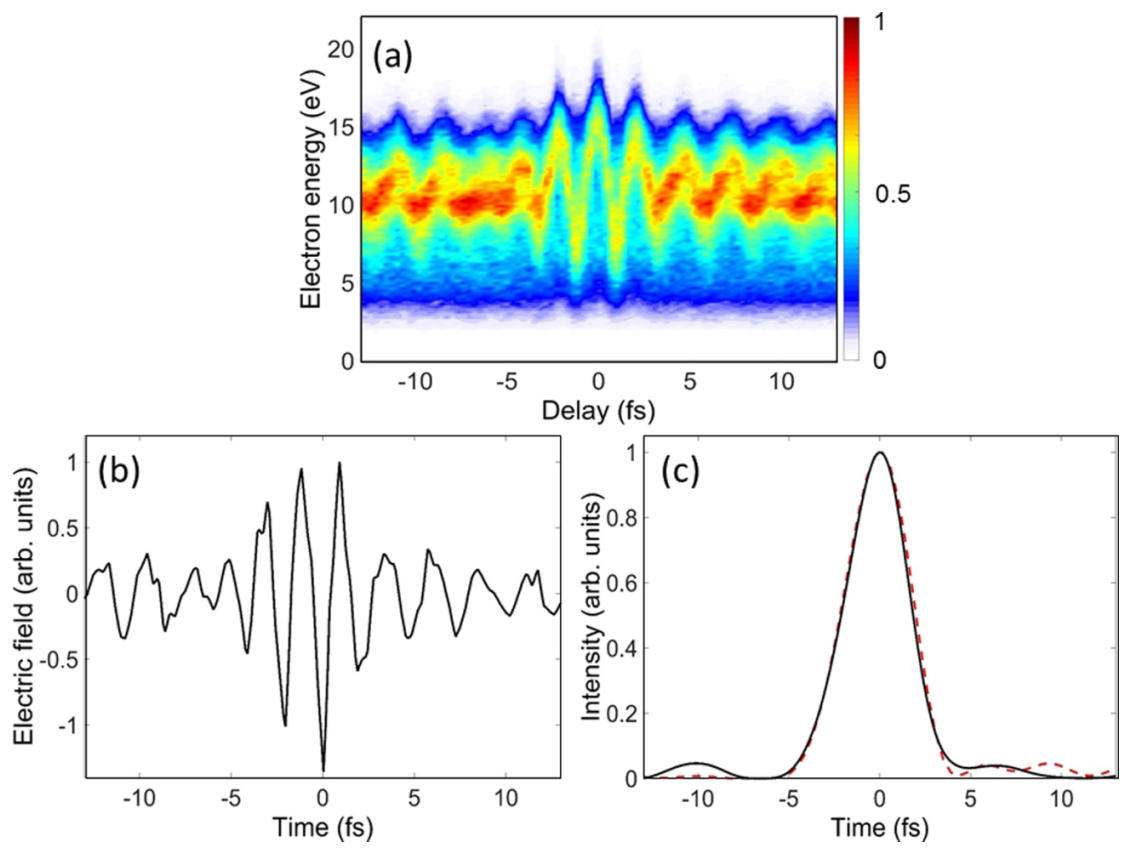

FIG. 3. (a) Streaking trace as a function of the temporal delay between the attosecond pulse and the streaking VIS/NIR pulse; temporal evolution of the electric field (b) and of the intensity profile (c) of the VIS/NIR pulse, reconstructed from the streaking trace (black solid line) and from the SRSI measurement (dashed red line). Electric field was calculated from the vector potential by discrete-derivative method and a moving average filter, with a 4-points span, was then applied. We calculated the intensity profile in the temporal domain by extracting the square envelope of the electric field via the Matlab-based "envelope" function, specified to the peaks of the field.

$$
\sigma_{T}=\left[\int_{-T / 2}^{T / 2} t^{2}|E(t)|^{2} d t\right]^{1 / 2} .
$$

The measured pulse spread was $\sigma_{T}=16.5 \mathrm{fs}\left(\sigma_{T}=10.3 \mathrm{fs}\right.$ for a transform-limited pulse). The energy ratio in a temporal window of \pm 4 fs (around the peak of the pulse) was measured to be $79 \%$ (94\% for a transform-limited pulse).

In order to cross-check the result obtained by the SRSI method, we have directly measured the electric field evolution of the pulses by using the attosecond streak-camera technique. $^{12,13}$ The basic idea of this experimental technique is to probe the temporal evolution of the electric field of a femtosecond pulse by using isolated attosecond pulses. As shown in Fig. 1, the beam transmitted through beam splitter BS was sent in an attosecond beamline. The visible/nearinfrared (VIS/NIR) beam was divided into two parts by using an ultrabroadband beam splitter. The transmitted pulses were used to produce XUV pulses by high-order harmonic generation (HHG) in an argon cell; isolated attosecond pulses (pulse duration $\sim 270$ as) were produced by employing the polarization gating technique. ${ }^{23,24}$ An aluminium filter was used to block the fundamental radiation and the low-order harmonics. The reflected part of the beam was collinearly recombined with the XUV pulses by using a mirror with a central hole. A pair of fused silica ultrathin wedges at Brewster angle was used to finely tune the dispersion compensation of the VIS/NIR pulses. The total amount of fused silica in the beam path was adjusted in order to measure the minimum pulse duration with the attosecond streak-camera setup. Of course, in this case, the dispersion optimization procedure was rather time consuming, since typical duration of a complete attosecond streak-camera measure was $\sim 10 \mathrm{~min}$.
The temporal delay between the VIS/NIR and XUV pulses was adjusted by using a piezoelectric translation stage. Both pulses were then collinearly focused into an electron time-of-flight spectrometer by using a toroidal mirror. The attosecond pulses ionize krypton by single-photon absorption in the presence of the streaking VIS/NIR pulse. The photoelectron spectra as a function of the delay between the attosecond and the VIS/NIR pulses, shown in Fig. 3(a), is shifted by $-\boldsymbol{A}\left(t_{i}\right)$, where $\boldsymbol{A}\left(t_{i}\right)$ is the vector potential of the VIS/NIR streaking pulse at the ionization instant. Therefore, the temporal evolution of $\boldsymbol{- A}(t)$ can be directly obtained by calculating the center of gravity of the streaking trace. The correspondent electric field, displayed in Fig. 3(b), was retrieved by performing a discrete-derivative calculation. The corresponding temporal evolution of the intensity profile was plotted as a function of time, as reported in Fig. 3(c), showing a 4 fs (FWHM) duration, in agreement with the result obtained by using the SRSI single-shot technique, which is reported in Fig. 3(c) as dashed line. The small differences in the wings of the pulse (delays $|\tau|<5$ fs) can be due to high-order dispersion terms not exactly matched in the SRSI and in the attosecond setups.

\section{CONCLUSION}

We demonstrated that self-referenced spectral interferometry can be implemented for complete temporal characterization of 4-fs pulses on a single-shot basis. A novel experimental setup was developed, characterized by low dispersion and collinear geometry. A dynamic range of $40 \mathrm{~dB}$ was demonstrated on a broad temporal window. The experimental results 
were in excellent agreement with the pulse reconstruction obtained by sampling the pulse electric field with isolated attosecond pulses.

\section{ACKNOWLEDGMENTS}

This work was supported by the European Research Council under the European Community's Seventh Framework Programme (No. FP7/2007-2013)/ERC Grant Agreement No. 227355 ELYCHE and by Laserlab-Europe (Grant Agreement No. 284464).

${ }^{1}$ D. J. Kane and R. Trebino, "Characterization of arbitrary femtosecond pulses using frequency-resolved optical gating," IEEE J. Quantum Electron. 29, 571-579 (1993).

${ }^{2}$ R. Trebino, Frequency-Resolved Optical Gating: The Measurement of Ultrashort Laser Pulses (Springer Science+Business Media, 2000).

${ }^{3}$ C. Iaconis and I. A. Walmsley, "Spectral phase interferometry for direct electric-field reconstruction of ultrashort optical pulses," Opt. Lett. 23, $792-794$ (1998).

${ }^{4}$ C. Dorrer, B. de Beauvoir, C. Le Blanc, S. Ranc, J.-P. Rousseau, P. Rousseau, and J.-P. Chambaret, "Single-shot real-time characterization of chirpedpulse amplification systems by spectral phase interferometry for direct electric-field reconstruction,” Opt. Lett. 24, 1644-1646 (1999).

${ }^{5}$ W. Kornelis, J. Biegert, J. W. G. Tisch, M. Nisoli, G. Sansone, C. Vozzi, S. De Silvestri, and U. Keller, "Single-shot kilohertz characterization of ultrashort pulses by spectral phase interferometry for direct electric-field reconstruction," Opt. Lett. 28, 281-283 (2003).

${ }^{6}$ T. Oksenhendler, S. Coudreau, N. Forget, V. Crozatier, S. Grabielle, R. Herzog, O. Gobert, and D. Kaplan, "Self-referenced spectral interferometry," Appl. Phys. B 99, 7-12 (2010).

${ }^{7}$ C. Dorrer and M. Joffre, "Characterization of the spectral phase of ultrashort light pulses,” C. R. Acad. Sci., Ser. IV: Phys. 2, 1415-1426 (2001).

${ }^{8}$ N. Minkovski, G. I. Petrov, S. M. Saltiel, O. Albert, and J. Etchepare, "Nonlinear polarization rotation and orthogonal polarization generation experienced in a single-beam configuration," J. Opt. Soc. Am. B 21, 1659-1664 (2004).

${ }^{9}$ L. Lepetit, G. Chériaux, and M. Joffre, "Linear techniques of phase measurement by femtosecond spectral interferometry for applications in spectroscopy," J. Opt. Soc. Am. B 12, 2467-2474 (1995).

${ }^{10}$ A. Jullien, L. Canova, O. Albert, D. Boschetto, L. Antonucci, Y.-H. Cha, J. P. Rousseau, P. Chaudet, G. Chériaux, J. Etchepare, S. Kourtev, N. Minkovski, and S. M. Saltiel, "Spectral broadening and pulse duration reduction during cross-polarized wave generation: Influence of the quadratic spectral phase," Appl. Phys. B 87, 595-601 (2007).

${ }^{11}$ A. Moulet, S. Grabielle, C. Cornaggia, N. Forget, and T. Oksenhendler, "Single-shot, high-dynamic-range measurement of sub-15 fs pulses by selfreferenced spectral interferometry," Opt. Lett. 35, 3856-3858 (2010).

${ }^{12}$ J. Itatani, F. Quéré, G. L. Yudin, M. Yu. Ivanov, F. Krausz, and P. B. Corkum, “Attosecond streak-camera,” Phys. Rev. Lett. 88, 173903 (2002).

${ }^{13}$ E. Goulielmakis, M. Uiberacker, R. Kienberger, A. Baltuška, V. Yakovlev, A. Scrinzi, Th. Westerwalbesloh, U. Kleineberg, U. Heinzmann, M. Drescher, and F. Krausz, "Direct measurement of light waves," Science $\mathbf{3 0 5}$ 1267-1269 (2004)

${ }^{14}$ M. Nisoli, S. De Silvestri, and O. Svelto, "Generation of high energy 10 fs pulses by a new pulse compression technique," Appl. Phys. Lett. 68, 2793-2795 (1996).

${ }^{15}$ M. Nisoli, S. De Silvestri, O. Svelto, R. Szipöcs, K. Ferencz, Ch. Spielmann, S. Sartania, and F. Krausz, "Compression of high energy laser pulses below 5 fs," Opt. Lett. 22, 522-524 (1997).

${ }^{16}$ A. Suda, M. Hatayama, K. Nagasaka, and K. Midorikawa, "Generation of sub-10-fs, 5-mJ-optical pulses using a hollow fiber with a pressure gradient," Appl. Phys. Lett. 86, 111116 (2005).

${ }^{17}$ F. Lücking, A. Trabattoni, S. Anumula, G. Sansone, F. Calegari, M. Nisoli, T. Oksenhendler, and G. Tempea, "In situ measurement of nonlinear carrier-envelope phase changes in hollow fiber compression," Opt. Lett. 39, 2302-2305 (2014).

${ }^{18}$ C. Vozzi, F. Calegari, F. Ferrari, M. Lucchini, S. De Silvestri, O. Svelto, G. Sansone, S. Stagira, and M. Nisoli, "Advances in laser technology for isolated attosecond pulse generation,” Laser Phys. Lett. 6, 259-267 (2009).

${ }^{19}$ G. Sansone, E. Benedetti, F. Calegari, C. Vozzi, L. Avaldi, R. Flammini, L. Poletto, P. Villoresi, C. Altucci, R. Velotta, S. Stagira, S. De Silvestri, and M. Nisoli, "Isolated single-cycle attosecond pulses," Science 314, 443-446 (2006).

${ }^{20}$ F. Calegari, D. Ayuso, A. Trabattoni, L. Belshaw, S. De Camillis, S. Anumula, F. Frassetto, L. Poletto, A. Palacios, P. Decleva, J. Greenwood, F. Martín, and M. Nisoli, "Ultrafast electron dynamics in phenylalanine initiated by attosecond pulses," Science 346, 336-339 (2014).

${ }^{21} \mathrm{C}$. Dorrer, "Influence of the calibration of the detector on spectral interferometry," J. Opt. Soc. Am. B 16, 1160-1168 (1999).

${ }^{22}$ T. Oksenhendler, "Self-referenced spectral interferometry theory," e-print arXiv:1204.4949 (2012).

${ }^{23}$ O. Tcherbakoff, E. Mével, D. Descamps, J. Plumridge, and E. Constant, "Time-gated high-order harmonic generation," Phys. Rev. A 68, 0438041-043804-4 (2003).

${ }^{24}$ I. J. Sola, E. Mével, L. Elouga, E. Constant, V. Strelkov, L. Poletto, P. Villoresi, E. Benedetti, J.-P. Caumes, S. Stagira, C. Vozzi, G. Sansone, and M. Nisoli, "Controlling attosecond electron dynamics by phase-stabilized polarization gating," Nat. Phys. 2, 319-322 (2006). 